Brief paper

\title{
Stability analysis for multi-agent systems using the incidence matrix: Quantized communication and formation control ${ }^{\text {W }}$
}

\author{
Dimos V. Dimarogonas ${ }^{\mathrm{a}, *}$, Karl H. Johansson ${ }^{\mathrm{b}}$ \\ a Laboratory for Information and Decision Systems, Massachusetts Institute of Technology, Cambridge, MA 02139, USA \\ ${ }^{\mathrm{b}}$ ACCESS Linnaeus Center, School of Electrical Engineering, Royal Institute of Technology, SE-100 44, Stockholm, Sweden
}

\section{A R T I C L E I N F O}

\section{Article history:}

Received 14 February 2009

Received in revised form

30 November 2009

Accepted 4 January 2010

Available online 21 February 2010

Keywords:

Multi-agent systems

Formation control

Quantized control

Algebraic graph theory

Networked control

\begin{abstract}
A B S T R A T
The spectral properties of the incidence matrix of the communication graph are exploited to provide solutions to two multi-agent control problems. In particular, we consider the problem of state agreement with quantized communication and the problem of distance-based formation control. In both cases, stabilizing control laws are provided when the communication graph is a tree. It is shown how the relation between tree graphs and the null space of the corresponding incidence matrix encode fundamental properties for these two multi-agent control problems.
\end{abstract}

(C) 2010 Elsevier Ltd. All rights reserved.

\section{Introduction}

The spectral properties of the Laplacian matrix of a graph were extensively used recently to provide convergence results in various multi-agent control problems (Arcak, 2007; Cortes, Martinez, \& Bullo, 2006; Olfati-Saber \& Murray, 2004; Olfati-Saber \& Shamma, 2005). In this paper we use another matrix namely, the incidence matrix and its spectral properties, in order to study the convergence properties of two multi-agent control problems. Cycles are not captured by the properties of the Laplacian, but note instead that the incidence matrix has an empty null space when the communication graph is a tree. This property is used to show that multi-agent networks represented by trees can compensate

\footnotetext{
This work was done within the TAIS-AURES project, which was supported by the Swedish Governmental Agency for Innovation Systems (VINNOVA) and Swedish Defence Materiel Administration (FMV). It was also supported by the Swedish Research Council, the Swedish Foundation for Strategic Research, and the EU NoE HYCON. Preliminary versions of this work appeared in the 2008 American Control Conference, Seattle, Washington, USA, June 11-13, 2008, and the 47th IEEE Conference on Decision and Control, December 9-11, 2008. Fiesta Americana Grand Coral Beach, Cancun, Mexico. This paper was recommended for publication in revised form by Associate Editor Murat Arcak under the direction of Editor Andrew R. Teel.

* Corresponding author. Tel.: +1 617324 0095; fax: +1 6172585779. E-mail addresses: ddimar@mit.edu (D.V.Dimarogonas), kallej@ee.kth.se (K.H. Johansson)
}

for bounded disturbances in the control input. On the other hand, in a cyclic graph, the error never ceases to propagate in these cycles. These facts are encoded by the definiteness properties of the quadratic form of the incidence matrix. The first problem to which we apply the properties of the incidence matrix is multi-agent state agreement under quantized communication. The only information each agent has is a quantized estimate of its neighbors' relative positions. We first treat a static communication topology and show that convergence is achieved in the case of a tree topology. The results are then extended to switching topologies. While results for discrete-time systems appeared recently (Carli, Fagnani, \& Zampieri, 2006; Johansson, Speranzon, \& Zampieri, 2005; Kashyap, Basar, \& Srikant, 2007), a continuous-time model is considered here. The second problem we consider is distance-based formation control. Such formations have been studied in the context of graph rigidity (Baillieul \& Suri, 2004; Hendrickx, Anderson, \& Blondel, 2005), where a common factor is the lack of globally stabilizing formation control laws. We propose here a control law that is based on the negative gradient of a potential function between each of the pairs of agents that form an edge in the formation graph. We show that the corresponding control law stabilizes the system to the desired formation provided that the graph is a tree. A similar result for directed acyclic graphs with three agents appeared in Cao, Anderson, Morse, and Yu (2008). We then show that it is necessary with a tree for stabilization to the desired formation.

The rest of the paper is organized as follows: preliminaries and the system model are discussed in Section 2. Section 3 treats the 
quantized agreement problem while Section 4 deals with distancebased formation control. A summary is given in Section 5 .

\section{Preliminaries}

\subsection{Graph theory}

We first review some elements of algebraic graph theory (Godsil \& Royle, 2001) used in the sequel. For an undirected graph $G=$ $(V, E)$ with $N$ vertices $V=\{1, \ldots, N\}$ and edges $E \subset V \times V$, the adjacency matrix $A=A(G)=\left(a_{i j}\right)$ is the $N \times N$ matrix given by $a_{i j}=1$, if $(i, j) \in E$, and $a_{i j}=0$, otherwise. If $(i, j) \in E$, then $i, j$ are adjacent. A path of length $r$ from $i$ to $j$ is a sequence of $r+1$ distinct vertices starting with $i$ and ending with $j$ such that consecutive vertices are adjacent. For $i=j$, this path is a cycle. If there is a path between any two vertices of $G$, then $G$ is connected. A connected graph is a tree if it contains no cycles. The degree $d_{i}$ of vertex $i$ is given by $d_{i}=\sum_{j} a_{i j}$. Let $\Delta=\operatorname{diag}\left(d_{1}, \ldots, d_{N}\right)$. The Laplacian of $G$ is the symmetric positive semidefinite matrix $L=$ $\Delta-A$. For a connected graph, $L$ has a single zero eigenvalue with the corresponding eigenvector $\mathbf{1}=[1, \ldots, 1]^{\mathrm{T}}$. An orientation on $G$ is the assignment of a direction to each edge. The incidence matrix $B=B(G)=\left(b_{i j}\right)$ of an oriented graph is the $\{0, \pm 1\}$ matrix with rows and columns indexed by the vertices and edges of $G$, respectively, such that $b_{i j}=1$ if the vertex $i$ is the head of the edge $j, b_{i j}=-1$ if the vertex $i$ is the tail of the edge $j$, and $b_{i j}=0$ otherwise. We have $L=B B^{\mathrm{T}}$. If $G$ contains cycles, the edges of each cycle have a direction, where each edge is directed towards its successor according to the cyclic order. A cycle $C$ is represented by a vector $v_{C}$ with $M=|E|$ elements. For each edge, the corresponding element of $v_{C}$ is equal to 1 if the direction of the edge with respect to $C$ coincides with the orientation assigned to the graph for defining $B$, and -1 , if the direction with respect to $C$ is opposite to the orientation. The elements corresponding to edges not in $C$ are zero. The cycle space of $G$ is the subspace spanned by vectors representing cycles in $G$ (Guattery \& Miller, 2000).

Let $x=\left[x_{1}, \ldots, x_{N}\right]^{\mathrm{T}}$, where $x_{i}$ is a real scalar variable assigned to vertex $i$ of $G$. Denote by $\bar{x}$ the $M$-dimensional stack vector of relative differences of pairs of agents that form an edge in $G$, where $M=|E|$ is the number of edges, in agreement with a defined orientation. In particular, denoting by $e_{i}=\left(h_{i}, t_{i}\right) \in E, i=$ $1, \ldots, M$, the edges of $G$, where $h_{i}, t_{i}$ are the head and tail of $e_{i}$ respectively, we denote $\bar{x}_{e_{i}}=x_{h_{i}}-x_{t_{i}}$. The vector $\bar{x}$ is given by $\bar{x}=\left[\bar{x}_{e_{1}}, \ldots, \bar{x}_{e_{M}}\right]^{\mathrm{T}}$. It is easy to verify that $L x=B \bar{x}$ and $\bar{x}=B^{\mathrm{T}} x$. For $\bar{x}=0$ we have that $L x=0$.

Lemma 1. If $G$ is a tree, then $B^{\mathrm{T}} B$ is positive definite.

Proof. For any $y \in \mathbb{R}^{M}$, we have $y^{\mathrm{T}} B^{\mathrm{T}} B y=|B y|^{2}$ and hence $y^{\mathrm{T}} B^{\mathrm{T}} B y>0$ if and only if $B y \neq 0$, i.e., the matrix $B$ has empty null space. For a connected graph, the cycle space of the graph coincides with the null space of $B$ (Lemma 3.2 in Guattery \& Miller, 2000 ). Thus, for $G$ with no cycles, zero is not an eigenvalue of $B$. This implies that $B^{\mathrm{T}} B$ is positive definite. $\diamond$

\subsection{Stability of a linear system}

Let $z=\left[z_{1}, \ldots, z_{M}\right]^{\mathrm{T}}$ denote a vector of real variables assigned to each edge of $G$. We examine the behavior of the system:

$\dot{z}=-B^{\mathrm{T}} B(z+e)$,

where $e$ is a state error to be defined in the sequel. For $F(z)=\frac{1}{2} z^{\mathrm{T}} z$, we have $\dot{F}(z)=-z^{\mathrm{T}} B^{\mathrm{T}} B z-z^{\mathrm{T}} B^{\mathrm{T}} B e$. If $G$ is a tree, then by Lemma 1

$\dot{F}(z) \leq-\lambda_{\min }\left(B^{\mathrm{T}} B\right)|z|^{2}+|z|\left\|B^{\mathrm{T}} B\right\||e|$.

We can now state the following result.
Lemma 2. Consider system (1) and assume that $G$ is a tree. Then,

- if $|e| \leq \Theta$, for some $\Theta>0$, then $z$ converges to a ball of radius $\frac{\left\|B^{\mathrm{T}} B\right\| \Theta}{\lambda_{\min }\left(B^{\mathrm{T}} B\right)}$ in finite time;

- if $|e| \leq \theta|z|$, for some $\theta>0$, then $z$ converges exponentially to the origin, provided that $\theta<\frac{\lambda_{\min }\left(B^{\mathrm{T}} B\right)}{\left\|B^{\mathrm{T}} B\right\|}$.

Proof. For $|e| \leq \Theta$, (2) yields $\dot{F}(z) \leq-\lambda_{\min }\left(B^{\mathrm{T}} B\right)|z|(|z|-$ $\left.\frac{\left\|B^{\mathrm{T}} B\right\| \Theta}{\lambda_{\min }\left(B^{\mathrm{T}} B\right)}\right)$ so the first statement follows. For the second, $|e| \leq$ $\theta|z|$ gives $\dot{F}(z) \leq-|z|^{2}\left(\lambda_{\min }\left(B^{\mathrm{T}} B\right)-\left\|B^{\mathrm{T}} B\right\| \theta\right)$ which is negative definite for $\theta<\frac{\lambda_{\min }\left(B^{\mathrm{T}} B\right)}{\left\|B^{\mathrm{T}} B\right\|}$. $\diamond$

Consider now instead the system

$\dot{z}=-B^{\mathrm{T}} B W z$,

where $W=\operatorname{diag}\left(w_{1}, \ldots, w_{M}\right)$ with $w_{j} \geq 0$. Note that (3) is a special case of (1) if $W z-z \equiv e$. The particular structure of (3) will be useful in the study of distance-based formation control.

Lemma 3. Consider system (3) and assume that $G$ is a tree. Then $z$ converges to the set $\left\{z \in \mathbb{R}^{M}: w_{i} z_{i}=0, \forall i=1, \ldots, M\right\}$.

Proof. Since $B^{\mathrm{T}} B$ is positive definite and $W$ is diagonal positive semidefinite, the linear system (3) is stable. At steady state we have $B^{\mathrm{T}} B W z=0$, and since $B^{\mathrm{T}} B$ is positive definite due to $G$ being a tree, we get $\left(B^{\mathrm{T}} B\right)^{-1} B^{\mathrm{T}} B W z=W z=0$ at steady state. The result follows from $W$ being diagonal. $\diamond$

We note that $B^{\mathrm{T}} B$ is defined as the "Edge Laplacian" in Zelazo, Rahmani, and Mesbahi (2007) and its properties are used for providing another perspective to the agreement problem.

\subsection{Multi-agent control system}

Consider $N$ agents. Let $q_{i} \in \mathbb{R}^{2}$ denote the position of agent $i$. Let $x_{i}, y_{i}$ denote the coordinates of agent $i$ in the $x$ and $y$ directions, respectively. Let $q=\left[q_{1}^{\mathrm{T}}, \ldots, q_{N}^{\mathrm{T}}\right]^{\mathrm{T}}$ denote the vector of all agents' positions. We assume that agents' motion obeys the single integrator model:

$\dot{q}_{i}=u_{i}, \quad i \in V=\{1, \ldots, N\}$,

where $u_{i}$ denotes the control input for each agent. We assume that each agent has limited information on the states and goals of the other group members. In particular, each agent is assigned a neighbor set $\mathcal{N}_{i} \subset V$, which is given by the agents with whom it can communicate.

\section{Quantized agreement}

The first problem we consider is agreement with quantized communication. We assume that agents aim to converge to a common value in the state space under quantized relative position information of their neighbors. It will be shown that the matrix $B^{\mathrm{T}} B$ plays an important role in the convergence of the system. Three classes of communication graphs are considered.

\subsection{Quantized control}

Consider system (4) in the $x$-direction and let $x=\left[x_{1}, \ldots, x_{N}\right]^{\mathrm{T}}$. Without loss of generality, we omit the notation regarding the $x$-direction from the control input. We then have $\dot{x}_{i}=u_{i}$. We consider the agreement control laws in Fax and Murray (2002) and Olfati-Saber and Murray (2004), which were given by 
$u_{i}=-\sum_{j \in \mathcal{N}_{i}}\left(x_{i}-x_{j}\right)$. The closed-loop nominal system (without quantization) is then given by $\dot{x}_{i}=-\sum_{j \in \mathcal{N}_{i}}\left(x_{i}-x_{j}\right), i \in V$, so that $\dot{x}=-L x$. Then, $\dot{\bar{x}}=B^{\mathrm{T}} \dot{\dot{x}}=-B^{\mathrm{T}} L x=-B^{\mathrm{T}} B \bar{x}$. Hence the nominal system is also given by $\dot{\bar{x}}=-B^{\mathrm{T}} B \bar{x}$.

In this section, each agent $i$ is assumed to have quantized measurements $Q\left(x_{i}-x_{j}\right), Q\left(y_{i}-y_{j}\right)$ of all $j \in \mathcal{N}_{i}$ where $Q():. \mathbb{R} \rightarrow$ $\mathbb{R}$ is the quantization function. Since the values of the quantizer are decomposed into the measurements $Q\left(x_{i}-x_{j}\right), Q\left(y_{i}-y_{j}\right)$ in the $x$ - and $y$-coordinates respectively, we can treat only the behavior of the system in the $x$-coordinates. The analysis that follows holds mutatis mutandis in the $y$-coordinates. We hence examine the stability properties of the closed-loop system in the $x$-coordinates under quantization, namely of the system $\dot{x}_{i}=$ $-\sum_{j \in \mathcal{N}_{i}} Q\left(x_{i}-x_{j}\right)$, with $i \in V$. Two classes of quantized sensors are considered: uniform and logarithmic quantizers. For a given $\delta_{u}>0$, a uniform quantizer $Q_{u}: \mathbb{R} \rightarrow \mathbb{R}$ satisfies $\left|Q_{u}(a)-a\right| \leq$ $\delta_{u}, \forall a \in \mathbb{R}$. For a given $\delta_{l}>0$, a logarithmic quantizer $Q_{l}: \mathbb{R} \rightarrow \mathbb{R}$ satisfies $\left|Q_{l}(a)-a\right| \leq \delta_{l}|a|, \forall a \in \mathbb{R}$. We use the notation $Q$ when we need not specify if it is a uniform or a logarithmic quantizer. For a vector $v=\left[v_{1}, \ldots, v_{d}\right]^{\mathrm{T}} \subset \mathbb{R}^{d}$ of size $d$, we define $Q_{u}(v) \triangleq$ $\left[Q_{u}\left(v_{1}\right), \ldots, Q_{u}\left(v_{d}\right)\right]^{\mathrm{T}}$ and $Q_{l}(v) \triangleq\left[Q_{l}\left(v_{1}\right), \ldots, Q_{l}\left(v_{d}\right)\right]^{\mathrm{T}}$. The following bounds also hold: $\left|Q_{u}(v)-v\right| \leq \delta_{u} \sqrt{d},\left|Q_{l}(v)-v\right| \leq$ $\delta_{l}|v|$.

\subsection{Static communication graph}

We first assume that the communication graph is static, i.e., that $\mathcal{N}_{i}$ do not vary over time. In the case of quantized information we have $\dot{x}_{i}=-\sum_{j \in \mathcal{N}_{i}} Q\left(x_{i}-x_{j}\right)$. Since $Q(-a)=-Q(a)$ for all $a \in \mathbb{R}$, we get

$\dot{\bar{x}}=-B^{\mathrm{T}} B Q(\bar{x})$,

where $Q(\bar{x})$ is the stack vector of all pairs $Q\left(x_{i}-x_{j}\right)$ with $(i, j) \in E$. The system (5) can be written in the form (1): $\dot{\bar{x}}=-B^{\mathrm{T}} B(\bar{x}+e)$ with $e \equiv Q(\bar{x})-\bar{x}$.

Consider now the quadratic edge function

$F(\bar{x})=\frac{1}{2} \bar{x}^{\mathrm{T}} \bar{x}$

Note that $\bar{x}=0$ guarantees that $x$ has all its elements equal, in the case of a connected graph. This is due to that $\bar{x}=0, L x=0$, which implies $x_{1}=x_{2}=\cdots=x_{N}$ for a connected graph. The following result is now a straightforward consequence of Lemma 2 :

Theorem 4. Assume that $G$ is static and a tree. Then system (5) has the following convergence properties.

- When $Q=Q_{u}, x$ converges to a ball of radius $\frac{\left\|B^{\mathrm{T}} B\right\| \delta_{u} \sqrt{M}}{\lambda_{\min }\left(B^{\mathrm{T}} B\right)}$ which is centered at the desired agreement equilibrium $x_{1}=x_{2}=\cdots=$ $x_{N}$ in finite time.

- When $Q=Q_{1}, x$ converges exponentially to the desired agreement equilibrium $x_{1}=x_{2}=\cdots=x_{N}$, provided that $\delta_{l}$ satisfies

$$
\delta_{l}<\frac{\lambda_{\min }\left(B^{\mathrm{T}} B\right)}{\left\|B^{\mathrm{T}} B\right\|} .
$$

From the previous analysis, for the case of a logarithmic quantizer we can compute

$$
\dot{F}(\bar{x}) \leq-|\bar{x}|^{2}\left(\lambda_{\min }\left(B^{\mathrm{T}} B\right)-\left\|B^{\mathrm{T}} B\right\| \delta_{l}\right) \text {. }
$$

By applying the Comparison Lemma, we get the following estimates of the convergence rate for the case of a logarithmic quantizer and a tree structure:

$F(\bar{x}(t)) \leq \mathrm{e}^{-2\left(\lambda_{\min }\left(B^{\mathrm{T}} B\right)-\left\|B^{\mathrm{T}} B\right\| \delta_{l}\right) t} F(\bar{x}(0))$ so that $|\bar{x}(t)| \leq \mathrm{e}^{-\left(\lambda_{\min }\left(B^{\mathrm{T}} B\right)-\left\|B^{\mathrm{T}} B\right\| \delta_{l}\right) t}|\bar{x}(0)|$ for all times $t \geq 0$. Using (8) we also get the following relations for the trajectories of the closed loop system in the case when the graph is not necessarily a tree:

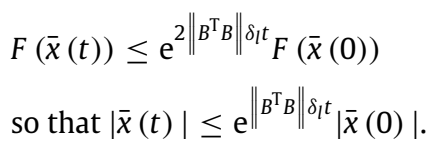

\subsection{Time-varying communication graph}

We next treat the case when the communication graph is timevarying. It is not possible to use $F(\bar{x})=\frac{1}{2} \bar{x}^{T} \bar{x}$ as a common Lyapunov function for the switched system, since $\bar{x}$ changes discontinuously whenever edges are added or deleted when the topology changes. We use instead $W=\max \left\{x_{1}, \ldots, x_{N}\right\}-$ $\min \left\{x_{1}, \ldots, x_{N}\right\}$ as a common Lyapunov function. Denote $x_{\max }=$ $x_{m_{1}}, x_{\min }=x_{m_{2}}$ where $m_{1} \triangleq \max _{i}\left\{i: x_{i}=\max _{k}\left\{x_{k}\right\}\right\}$, and $m_{2} \triangleq \min _{i}\left\{i: x_{i}=\min _{k}\left\{x_{k}\right\}\right\}$. With this definition, the system is guaranteed not to exhibit Zeno behavior (Lygeros, Johansson, Simic, Zhang, \& Sastry, 2003). This is due to that if there exists an interval $[\tau, \tau+\Delta \tau]$ with $\Delta \tau>0$, for which there exist two or more agents that simultaneously attain the maximum (minimum) value, then only the agent with the largest (smallest) index is considered. The notation $\mathcal{T}=\left\{t_{1}, t_{2} \ldots,\right\}$ is used for the set of switching instants, i.e., times when a new link is created or an existing one is lost, or the maximum or minimum element changes, i.e., a new agent attains the maximum or minimum value, $x_{\max }$ or $x_{\min }$, respectively. We will use the extension of LaSalle's Invariance Principle for hybrid systems (Lygeros et al., 2003) to check the stability of the overall system. The main result is stated as the following theorem.

Theorem 5. Assume that the time-varying communication graph $G=G(t)$ remains a tree for all intervals $\left[t_{p}, t_{p+1}\right]$ and the quantizer is logarithmic. Further assume that the gain of the quantizer $\delta_{l}$ satisfies

$\delta_{l}<\min _{B \in T(B)} \frac{\lambda_{\min }\left(B^{\mathrm{T}} B\right)}{\left\|B^{\mathrm{T}} B\right\|}$,

where the minimization is over all possible incidence matrices that belong to the set $T(B)$ of incidence matrices corresponding to all possible trees with $N$ vertices. Then, $x$ converges to an agreement point $x_{1}=x_{2}=\cdots=x_{N}$.

Proof. We show that $W$ is strictly decreasing in between switching instances. For the logarithmic quantizer, we have $\operatorname{sign}\left(Q_{l}(x)\right)=$ $\operatorname{sign}(x)$. Since $x_{\max } \geq x_{i} \geq x_{\min }$ for all $i \in V$, the following equations hold for all $t \in\left[t_{p}, t_{p+1}\right],\left[t_{p}, t_{p+1}\right] \in \mathcal{T}: \dot{x}_{\max }=$ $-\sum_{j \in \mathcal{N}_{\max }} Q_{l}\left(x_{\max }-x_{j}\right) \leq 0$, and $\dot{x}_{\min }=-\sum_{j \in \mathcal{N}_{\max }} Q_{l}\left(x_{\min }-x_{j}\right)$ $\geq 0$. Thus, $W$ is non-increasing throughout the closed loop system evolution. We now show that $W$ is strictly decreasing within each subinterval $[\tau, \tau+\Delta \tau]$ of $\left[t_{p}, t_{p+1}\right]$ with $\Delta \tau>0$ as long as the graph is a tree and the system has not reached an agreement point $\bar{x}=0$. This is proved by contradiction. Assume first that $x_{\max }$ is constant at each time instant of the time interval in consideration, i.e. $\dot{x}_{\max }=0$, for all $t \in[\tau, \tau+\Delta \tau]$. This is equivalent to $\sum_{j \in \mathcal{N}_{\max }} Q_{l}\left(x_{\max }-x_{j}\right)=0$, and since $x_{\max } \geq x_{i}$ for all $i \in\{1, \ldots, N\}$, the latter implies that $x_{j}=x_{\max }$ for all $j \in \mathcal{N}_{\text {max }}$. Pick any $k \in \mathcal{N}_{\text {max }}$, where $k$ does not coincide with the maximum vertex. Then $x_{k} \geq x_{j}$, for all $j \in \mathcal{N}_{k}$ and hence $\dot{x}_{k}=-\sum_{j \in \mathcal{N}_{k}} Q_{l}\left(x_{k}-x_{j}\right) \leq 0$. If $\dot{x}_{k}<0$, then necessarily $\dot{x}_{\max }<0$ since $x_{k}=x_{\max }$ for all $t \in[\tau, \tau+\Delta \tau]$. Hence we also have $\dot{x}_{k}=0$ and hence $x_{j}=x_{k}=x_{\max }$ for all $j \in \mathcal{N}_{k}$. We can now repeat the same procedure for a random $l \in \mathcal{N}_{k}$. Since the graph is a tree and has finite number of vertices, we 
conclude that there exist a finite number of iterations of the above procedure that propagates to every vertex in the graph. Thus, all vertices in the graph should have a zero time derivative. By virtue of the above procedure all vertices then will have a common value equal to the constant maximum value of $x_{\max }$. This is a contradiction to the fact that the function $F$ defined in (6) is strictly decreasing, by virtue of (8), (7) and (11), as long as the system has not reached agreement. We thus conclude that there should be at least one vertex $p$ chosen in the above iterative procedure which has a strictly negative time derivative at some $t \in[\tau, \tau+\Delta \tau]$. Since the above procedure suggests that $x_{p}=x_{\max }$, and therefore $\dot{x}_{p}=\dot{x}_{\max }$, for all $t \in[\tau, \tau+\Delta \tau]$, we conclude that $x_{\max }$ is strictly decreasing in $[\tau, \tau+\Delta \tau]$. The above analysis can be used to show-albeit not necessary for our proof-that $x_{\min }$ is strictly increasing in $[\tau, \tau+\Delta \tau]$. We conclude that $W$ strictly decreases within each time interval $\left[t_{p}, t_{p+1}\right]$, i.e., $W\left(t_{p}\right)<W\left(t_{p+1}\right)$, and thus, $W$ converges to zero as $t \rightarrow \infty$. The latter corresponds to a desired agreement point by definition. This completes the proof. $\diamond$

\subsection{Loss of connectivity}

The above result is useful when the communication graph retains the tree structure at all switching instances. A different case occurs if we allow for the tree assumption to be lost for some times. In particular, we assume that in between moments where the team switches to a different tree, there are time intervals when the communication graph is not a tree. Hence we consider a switching sequence of the form $\mathcal{T}=\left\{0=t_{01}, t_{1}, t_{12}, t_{2}, t_{23}, t_{3}, \ldots\right\}$, where intervals of the form $\Delta t_{p}=t_{p}-t_{p-1, p}>0$ correspond to a tree while the reset intervals $\Delta t_{p, p+1}=t_{p, p+1}-t_{p}>0$ correspond to a switch between two trees. The connectivity and tree assumptions may not hold in the reset intervals $\Delta t_{p, p+1}$. We assume that each $\Delta t_{p}$ where the topology is a tree has a minimum dwell time $\Delta t_{\mathrm{min}}$, i.e., $\Delta t_{p}>\Delta t_{\min }$. The following result states that agreement can still be achieved provided that the reset intervals are small enough.

Theorem 6. Assume that the time-varying communication graph $G=G(t)$ is a tree for all time intervals $\Delta t_{p}=t_{p}-t_{p-1, p}$ and the quantizer is logarithmic. Further assume that there is a path connecting the maximum and the minimum vertex, for all reset time intervals of the form $\Delta t_{p, p+1}=t_{p, p+1}-t_{p}$. Assume that there exists an $\varepsilon$, where $0<\varepsilon<\min _{B \in T(B)} \frac{\lambda_{\min }\left(B^{\mathrm{T}} B\right)}{\left\|B^{\mathrm{T}} B\right\|}$, such that the quantizer gain satisfies $\delta_{l}<\min _{B \in T(B)} \frac{\lambda_{\min }\left(B^{\mathrm{T}} B\right)}{\left\|B^{\mathrm{T}} B\right\|}-\varepsilon$. Furthermore, assume that the tree time intervals $\Delta t_{p}$ satisfy $\Delta t_{\min }>\frac{2 \ln (N(N-1) / 2)}{\varepsilon \cdot \max _{B \in T(B)}\left\|B^{T} B\right\|}$. Then the closed-loop system converges to an agreement point $x_{1}=x_{2}=\cdots=$ $x_{N}$, provided that the reset time intervals $\Delta t_{p, p+1}$ satisfy $\Delta t_{p, p+1}<$ $\Delta t_{\max }^{r}=\min _{p} \frac{v_{p+1} \Delta t_{\min }-2 \ln (N(N-1) / 2)}{\left\|B_{p, p+1}^{\mathrm{T}} B_{p, p+1}\right\| \delta_{l}}$, where the minimization is held over all incidence matrices $B_{p, p+1}$ corresponding to graphs with $N$ vertices and $v_{p+1}=\lambda_{\min }\left(B_{p+1}^{\mathrm{T}} B_{p+1}\right)-\left\|B_{p+1}^{\mathrm{T}} B_{p+1}\right\| \delta_{l}$.

Proof. We consider $W_{c}=\frac{W}{\sqrt{N(N-1)}}$ as a common Lyapunov function for the overall switched system. Since for all intervals there is a path $m_{1}, l_{1}, l_{2}, \ldots, l_{f}, m_{2}$ connecting the maximum and minimum vertices, we have $W=x_{m_{1}}-x_{m_{2}}=x_{m_{1}}-$ $x_{l_{1}}+x_{l_{1}}-x_{l_{2}}+\cdots+x_{l_{f}}-x_{m_{2}}$, and using the inequality $n \sum_{i=1}^{n} r_{i}^{2} \geq\left(\sum_{i=1}^{n} r_{i}\right)^{2}, \forall r_{i} \in \mathbb{R}$, we have $W^{2} \leq \frac{N(N-1)}{2}$. $\left[\left(x_{m_{1}}-x_{l_{1}}\right)^{2}+\left(x_{l_{1}}-x_{l_{2}}\right)^{2} \ldots\left(x_{l_{f}}-x_{m_{2}}\right)^{2}\right] \leq N(N-1) F$, and hence $W_{c} \leq \sqrt{F}$ where $F=F(\bar{x})$ is the quadratic function (6) corresponding to the edges of $G(t)$ at each time instant and $N(N-$ $1) / 2$ is the maximum number of edges at each time instant. Hence the candidate common Lyapunov function is bounded from above by $F$ at each time instant, where $F=F(\bar{x})$ is the quadratic edge function corresponding to the vector $\bar{x}$ of edges at the same time instant. All pairs $i, j \in\{1, \ldots, N\}$ satisfy $\left|x_{\max }-x_{\min }\right| \geq\left|x_{i}-x_{j}\right|$ and thus, $\frac{M}{2}\left(x_{\max }-x_{\min }\right)^{2} \geq \frac{1}{2} \sum_{(i, j) \in E}\left(x_{i}-x_{j}\right)^{2}=F$. Since the maximum number of edges $M$ is $N(N-1) / 2$ the last equation implies $W \geq \frac{2}{\sqrt{N(N-1)}} \sqrt{F}$, so that $W_{c} \geq \frac{2}{N(N-1)} \sqrt{F}$. We hence have

$\frac{2}{N(N-1)} \sqrt{F} \leq W_{c} \leq \sqrt{F}$

for all possible quadratic edge functions $F$ corresponding either to a tree interval or a reset interval.

With a slight abuse of notation, denote by $F_{p}$ the quadratic edge function $F$ corresponding to a random tree that represents the communication topology in the time interval $\Delta t_{p}$ and by $F_{p, p+1}$ the quadratic edge function $F$ corresponding to the reset time interval $\Delta t_{p, p+1}$. For two consecutive intervals $\left[t_{p}, t_{p, p+1}\right],\left[t_{p, p+1}, t_{p+1}\right]$, using (9), (10) and (12) we have

$$
\begin{aligned}
W_{c} & \left(t_{p+1}\right) \leq \sqrt{F_{p+1}\left(t_{p+1}\right)} \\
\leq & \mathrm{e}^{-\left(\lambda_{\min }\left(B_{p+1}^{\mathrm{T}} B_{p+1}\right)-\left\|B_{p+1}^{\mathrm{T}} B_{p+1}\right\| \delta_{l}\right) \Delta t_{p+1}} \sqrt{F_{p+1}\left(t_{p, p+1}\right)} \\
\leq & \mathrm{e}^{-\left(\lambda_{\min }\left(B_{p+1}^{\mathrm{T}} B_{p+1}\right)-\left\|B_{p+1}^{\mathrm{T}} B_{p+1}\right\| \delta_{l}\right) \Delta t_{p+1}} \frac{N(N-1)}{2} W_{c}\left(t_{p, p+1}\right) \\
\leq & \mathrm{e}^{-\left(\lambda_{\min }\left(B_{p+1}^{\mathrm{T}} B_{p+1}\right)-\left\|B_{p+1}^{\mathrm{T}} B_{p+1}\right\| \delta_{l}\right) \Delta t_{p+1}} \\
& \times \frac{N(N-1)}{2} \sqrt{F_{p, p+1}\left(t_{p, p+1}\right)} \\
\leq & \mathrm{e}^{-\left(\lambda_{\min }\left(B_{p+1}^{\mathrm{T}} B_{p+1}\right)-\left\|B_{p+1}^{\mathrm{T}} B_{p+1}\right\| \delta_{l}\right) \Delta t_{p+1}} \frac{N(N-1)}{2} \\
& \times \mathrm{e}^{\left\|B_{p, p+1}^{\mathrm{T}} B_{p, p+1}\right\| \delta_{l} \Delta t_{p, p+1} \sqrt{F_{p, p+1}\left(t_{p}\right)}} \\
\leq & \left(\frac{N(N-1)}{2}\right)^{2} \mathrm{e}^{-\left(\lambda_{\min }\left(B_{p+1}^{\mathrm{T}} B_{p+1}\right)-\left\|B_{p+1}^{\mathrm{T}} B_{p+1}\right\| \delta_{l}\right) \Delta t_{p+1}} \\
& \times \mathrm{e}^{\left\|B_{p, p+1}^{\mathrm{T}} B_{p, p+1}\right\| \delta_{l} \Delta t_{p, p+1}} W_{c}\left(t_{p}\right)
\end{aligned}
$$

where, in accordance with the defined notation, $B_{p+1} \in T(B)$ is an incidence matrix belonging to the set $T(B)$ of incident matrices corresponding to trees with $N$ vertices, while $B_{p, p+1}$ is an arbitrary incidence matrix corresponding to a graph with $N$ vertices. It suffices to show that $W_{c}$ strictly decreases in $t_{p}, t_{p+1}$. This is equivalent to $-\left(\lambda_{\min }\left(B_{p+1}^{\mathrm{T}} B_{p+1}\right)-\left\|B_{p+1}^{\mathrm{T}} B_{p+1}\right\| \delta_{l}\right) \Delta t_{p+1}+$ $\left\|B_{p, p+1}^{\mathrm{T}} B_{p, p+1}\right\| \delta_{l} \Delta t_{p, p+1}<-2 \ln \left(\frac{N(N-1)}{2}\right)$. Using $\Delta t_{p+1}>\Delta t_{\min }$, an upper bound on the reset interval time for which the above inequality holds is given by $\Delta t_{p, p+1}<\frac{v_{p+1} \Delta t_{\min }-2 \ln (N(N-1) / 2)}{\left\|B_{p, p+1}^{\mathrm{T}} B_{p, p+1}\right\| \delta_{l}}$, where the parameter $v_{p+1}=\lambda_{\min }\left(B_{p+1}^{\mathrm{T}} B_{p+1}\right)-\left\|B_{p+1}^{\mathrm{T}} B_{p+1}\right\| \delta_{l}$ is always positive, due to $\delta_{l}$ satisfying $\delta_{l}<\min _{B \in T(B)} \frac{\lambda_{\min }\left(B^{\mathrm{T}} B\right)}{\left\|B^{\mathrm{T}} B\right\|}-$ $\varepsilon$. Due to the fact that $\Delta t_{\min }$ satisfies $\Delta t_{\min }>\frac{2 \ln (N(N-1) / 2)}{\varepsilon \cdot \max _{B \in T(B)}\left\|B^{\mathrm{T}} B\right\|}$, there is a strictly positive upper bound on the reset intervals $\Delta t_{\max }^{r}$ for which $-\left(\lambda_{\min }\left(B_{p+1}^{\mathrm{T}} B_{p+1}\right)-\left\|B_{p+1}^{\mathrm{T}} B_{p+1}\right\| \delta_{l}\right) \Delta t_{p+1}+$ $\left\|B_{p, p+1}^{\mathrm{T}} B_{p, p+1}\right\| \delta_{l} \Delta t_{p, p+1}<-2 \ln \left(\frac{N(N-1)}{2}\right)$ holds, i.e. we have $\Delta t_{p, p+1}<\Delta t_{\max }^{r}$ for all $p$, and $\Delta t_{\max }^{r}=\min _{p} \frac{v_{p+1} \Delta t_{\min }-2 \ln (N(N-1) / 2)}{\left\|B_{p, p+1}^{\mathrm{T}} B_{p, p+1}\right\| \delta_{l}}$. Hence for sufficiently small reset intervals, $W_{c}$ is strictly decreasing, i.e., $W_{c}\left(t_{p+1}\right)<W_{c}\left(t_{p}\right)$ for all $p$. The result follows by allowing $p$ go to infinity. $\diamond$ 


\section{Distance-based formation control}

The second multi-agent problem considered is distance-based formation stabilization. A formation is an assignment of scalar weights $d_{i j}=d_{j i}>0$ to each edge $(i, j) \in E$ of the communication graph $G$. These weights represent the distance to which agents $i, j$ should converge. Define the set $\Phi \triangleq\{q \in$ $\left.\mathbb{R}^{2 N} \mid\left\|q_{i}-q_{j}\right\|=d_{i j}, \forall(i, j) \in E\right\}$ of desired distance-based formations. The desired formation is called feasible if $\Phi$ is non-empty. The problem treated in this section is summarized as follows: derive control laws, for which the information available for each agent $i$ is encoded in $\mathcal{N}_{i}$ that drive the agents to the desired formation, i.e., $\lim _{t \rightarrow \infty} q(t)=q^{*} \in \Phi$.

\subsection{Control law and stability analysis}

Let $\beta_{i j}(q)=\left\|q_{i}-q_{j}\right\|^{2}$ for any $i, j \in V$. The class $\Gamma$ of formation potentials $\gamma \in \Gamma$ between agents $i, j, j \in \mathcal{N}_{i}$ is defined to have the following properties: (1) $\gamma: \mathbb{R}^{+} \rightarrow \mathbb{R}^{+} \cup\{0\}$ is a function of the distance between $i$ and $j$, i.e., $\gamma=\gamma\left(\beta_{i j}\right)$, (2) $\gamma\left(\beta_{i j}\right)$ is continuously differentiable, and (3) $\gamma\left(d_{i j}^{2}\right)=0$ and $\gamma\left(\beta_{i j}\right)>0$ for all $\beta_{i j} \neq d_{i j}^{2}$. We also define $\rho_{i j} \triangleq \frac{\partial \gamma\left(\beta_{i j}\right)}{\partial \beta_{i j}}$. Note that $\rho_{i j}=\rho_{j i}$, for all $i, j \in V, i \neq j$. The proposed control law for $i \in V$ is

$u_{i}=-\sum_{j \in \mathcal{N}_{i}} \frac{\partial \gamma\left(\beta_{i j}(q)\right)}{\partial q_{i}}=-\sum_{j \in \mathcal{N}_{i}} 2 \rho_{i j}\left(q_{i}-q_{j}\right)$.

Let $\otimes$ denote the Kronecker product. Then (13) is written in stack vector form as $u=-2\left(R \otimes I_{2}\right) q$ where $u=\left[u_{1}^{\mathrm{T}}, \ldots, u_{N}^{\mathrm{T}}\right]^{\mathrm{T}}$ and the symmetric matrix $R$ is given by $R_{i j}=-\rho_{i j}$, for $j \in \mathcal{N}_{i}$, $R_{i j}=0$, for $j \notin \mathcal{N}_{i}$, and $R_{i i}=\sum_{j \in \mathcal{N}_{i}} \rho_{i j}$, for all $i \in V$. Let $V_{f}(q)=\sum_{i} \sum_{j \in \mathcal{N}_{i}} \gamma\left(\beta_{i j}(q)\right)$. Its gradient is $\nabla V_{f}=4\left(R \otimes I_{2}\right) q$, so that its time-derivative is given by

$\dot{V}_{f}=-8\left\|\left(R \otimes I_{2}\right) q\right\|^{2} \leq 0$.

The following theorem now holds.

Theorem 7. Assume that (4) evolves under (13), and that $G$ is connected. Then $u_{i}(t) \rightarrow 0$ as $t \rightarrow \infty$ for all $i \in V$, and the closed loop system is stable.

Proof. The level sets of $V_{f}$ are compact and invariant with respect to the relative positions of adjacent agents. Specifically, the set $\Omega_{c}=\left\{q: V_{f}(q) \leq c\right\}$ for $0<c<\infty$ is closed by continuity of $V_{f}$. From $V_{f} \leq c$ we have $\gamma\left(\beta_{i j}\right) \leq c$ for all $(i, j) \in E$. This implies that there is a $\xi$, where $0<\xi<\infty$, such that $\beta_{i j} \leq \xi$, by definition of $\Gamma$, and thus, $\left\|q_{i}-q_{j}\right\| \leq \sqrt{\xi}$ for all $(i, j) \in E$. Since the maximum length of the path between any two vertices of a connected graph is $N-1$, we have $0 \leq\left\|q_{i}-q_{j}\right\| \leq(N-1) \sqrt{\xi}$ for all $i, j \in V$. Eq. (14) and LaSalle's principle now guarantee that the system converges to the largest invariant subset of $S=\left\{q:\left(R(q) \otimes I_{2}\right) q=0\right\}$. Since $u=\dot{q}=-2\left(R \otimes I_{2}\right) q$, we have $u \rightarrow 0$ as $t \rightarrow \infty$ and the result follows. Compactness of $\Omega_{c}$ and (14) imply also that the closed loop system is stable. $\diamond$

We next provide a formation potential that guarantees formation stabilization for a class of communication graphs. In particular, we now consider:

$\gamma\left(\beta_{i j}(q)\right)=\frac{\left(\beta_{i j}-d_{i j}^{2}\right)^{2}}{\beta_{i j}}$.

Note that this potential satisfies all properties of the set $\Gamma$. Moreover, $\gamma\left(\beta_{i j}\right) \leq c \Rightarrow 0 \leq \frac{\left(\beta_{i j}-d_{i j}^{2}\right)^{2}}{\beta_{i j}} \leq c \Rightarrow 0 \leq\left(\beta_{i j}-d_{i j}^{2}\right)^{2} \leq$ $c \beta_{i j} \Rightarrow \beta_{i j} \in\left[\xi_{1}, \xi_{2}\right]$ where $\xi_{1,2}=\frac{1}{2}\left(2 d_{i j}^{2}+c \pm \sqrt{4 c d_{i j}^{2}+c^{2}}\right)$. For this case, $\rho_{i j}=\frac{\partial \gamma\left(\beta_{i j}\right)}{\partial \beta_{i j}}=\frac{\beta_{i j}^{2}-d_{i j}^{4}}{\beta_{i j}^{2}}$. For this potential, the following Lemma holds.

Lemma 8. Consider system (4) driven by (13) with $\gamma$ as in (15), and starting from a set of initial conditions $\ell(q)=\left\{q \mid\left\|q_{i}-q_{j}\right\|>\right.$ $0, \forall(i, j) \in E\}$. Then $\ell(q)$ is invariant for the trajectories of the closed loop system.

Proof. For every initial condition $q(0) \in \ell(q)$, the time derivative of $V_{f}$ remains non-positive for all $t \geq 0$, by virtue of (14). Hence $V_{f}(q(t)) \leq V_{f}(q(0))<\infty$ for all $t \geq 0$. When $\left\|q_{i}-q_{j}\right\| \rightarrow 0$ for at least one pair of agents $i$, $j$, with $j \in \mathcal{N}_{i}$, we have $V_{f}(q) \rightarrow \infty$, which is impossible. We conclude that $q(t) \in \ell(q)$, for all $t \geq 0$. $\diamond$

Thus, $\beta_{i j}(t)>0$, i.e., $q_{i}(t) \neq q_{j}(t)$, for all $t \geq 0$ and all $(i, j) \in E$. This will be used in the stability analysis of the closed-loop system. Denote by $\bar{q}$ the $M$-dimensional stack vector of relative position differences of pairs of agents that form an edge in $G$, where $M$ is the number of edges, i.e, $M=|E|$ and $\bar{q}=\left[\bar{q}_{1}^{\mathrm{T}}, \ldots, \bar{q}_{M}^{\mathrm{T}}\right]^{\mathrm{T}}$, where $\bar{q}_{e}=q_{i}-q_{j}$ for $e=(i, j) \in E$. Equation $\dot{q}=-2\left(R \otimes I_{2}\right) q$ implies

$\dot{\bar{q}}=-\left(B^{\mathrm{T}} B W_{f} \otimes I_{2}\right) \bar{q}$,

where $W_{f}=2 \cdot \operatorname{diag}\left\{\rho_{e}, e \in E\right\} \in \mathbb{R}^{M \times M}$. Note that (16) is in the form (3) in two dimensions. We then have the following theorem.

Theorem 9. Assume that (4) evolves under the control law (13) with $\gamma$ as in (15), and that the communication graph is a tree. Further assume that the desired formation is feasible, i.e., $\Phi \neq \varnothing$. Then the agents are driven to the desired formation, i.e., $\lim _{t \rightarrow \infty} q(t)=$ $q^{*} \in \Phi$.

Proof. Since at steady state, $\dot{q}=u=-2\left(R \otimes I_{2}\right) q=0$, we also have $\dot{\bar{q}}_{e}=0$ for all $e \in E$ and thus $\dot{\bar{q}}=0$. Eq. (16) yields $\left(B^{\mathrm{T}} B W_{f} \otimes I_{2}\right) \bar{q}=0$. By virtue of Lemma 3 the system converges to a configuration where $\rho_{e} \bar{q}_{e}=0$ for all $e \in E$. Since $\rho_{e}$ is scalar this implies $\rho_{e}=0$ or $\bar{q}_{e}=0$. However, for all $e \in E$ we have $\bar{q}_{e}(t) \neq 0$ for all $t \geq 0$, due to Lemma 8 . We conclude that $\rho_{e}=0$ for all $e \in E$ at steady state and hence $\beta_{i j}=d_{i j}^{2}$, i.e, $\left\|q_{i}-q_{j}\right\|=d_{i j}$ for all $(i, j) \in E$, since $\rho_{i j}=\frac{\beta_{i j}^{2}-d_{i j}^{4}}{\beta_{i j}^{2}}$. $\diamond$

\subsection{Tree communication graph is necessary}

We next characterize the communication graphs for which a control law of the form (13) leads to the desired formation for any choice of potential $\gamma \in \Gamma$. In particular, for any choice of $\gamma \in \Gamma$, the closed-system dynamics are given by $\dot{q}=u=-2\left(R \otimes I_{2}\right) q$, and thus by $\dot{\bar{q}}=-\left(B^{\mathrm{T}} B W_{f} \otimes I_{2}\right) \bar{q}$ in the edge space. The analysis leading to Theorem 9 guarantees that $\left(B^{\mathrm{T}} B W_{f} \otimes I_{2}\right) \bar{q}=0$ at a steady state. By virtue of Lemma 1 , the matrix $B^{\mathrm{T}} B$ is non-singular only when $G$ contains no cycles. The following result holds.

Theorem 10. Assume that the system (4) evolves under the control law (13) and that $\Phi$ is non-empty. Further assume that $G$ is connected. Then there exists a formation potential $\gamma \in \Gamma$ such that (i) $\dot{\bar{q}}=0$ only for $q \in \Phi$, and (ii) $\lim _{t \rightarrow \infty} q(t)=q^{*} \in \Phi$ hold, if and only if the graph $G$ is a tree.

Proof. The "if" part is shown in Theorem 9, with the choice of formation potential field (15). For the "only if" part, we know that the closed-loop system reaches a steady state at which $u=0$, by virtue of Theorem 7. This implies that $\dot{\bar{q}}=\left(B^{\mathrm{T}} B W_{f} \otimes I_{2}\right) \bar{q}=0$. We will show that condition (i) cannot hold if $G$ is not a tree. If $G$ is not a tree, then $B^{\mathrm{T}} B$ is singular and then the null space of $B$, and thus 
$B^{\mathrm{T}} B$, is nonempty. In fact, in this case, using properties of Kronecker products (Horn \& Johnson, 1996) we have $\left(B^{\mathrm{T}} B W_{f} \otimes I_{2}\right) \bar{q}=0$, so that $\left(B^{\mathrm{T}} B \otimes I_{2}\right)\left(W_{f} \otimes I_{2}\right) \bar{q}=0$. Multiplying by $\left(\bar{q}\left(W_{f} \otimes I_{2}\right)\right)^{\mathrm{T}}$ on the left-hand side, we get $\left(\bar{q}\left(W_{f} \otimes I_{2}\right)\right)^{\mathrm{T}}\left(B^{\mathrm{T}} B \otimes I_{2}\right)\left(W_{f} \otimes I_{2}\right)$ $\bar{q}=0$, which implies $\bar{q}^{\mathrm{T}}\left(B W_{f} \otimes I_{2}\right)^{\mathrm{T}}\left(B W_{f} \otimes I_{2}\right) \bar{q}=0$, so that $\left(B W_{f} \otimes I_{2}\right) \bar{q}=0$. Denoting by $\bar{x}, \bar{y}$ the stack vectors of the elements of $\bar{q}$ in the $x$ - and $y$-coordinates, the last equation yields $B W_{f} \bar{x}=B W_{f} \bar{y}=0$, i.e., $W_{f} \bar{x}, W_{f} \bar{y}$ belong to the null space of $B$. Since $G$ contains cycles, the null space of $B$ is non-empty. Thus we cannot reach the conclusion of the proof of Theorem 9 that $\left(W_{f} \otimes I_{2}\right) \bar{q}=0$. In fact, equations $B W_{f} \bar{x}=B W_{f} \bar{y}=0$ have an infinite number of solutions, since $B^{\mathrm{T}} B$ is now singular. Hence in this case, $\left(B^{\mathrm{T}} B W_{f} \otimes I_{2}\right) \bar{q}=0$ does not hold only when $q \in \Phi$, as was the case in Theorem 9. Thus (i) cannot hold if $G$ is not a tree. We conclude that (i) and (ii) hold only if $G$ is a tree. $\diamond$

The last result states that if $G$ contains cycles, then we cannot design a control law of the form (13) that stabilizes the agents to the desired relative distances.

\section{Conclusions}

We used the spectral properties of the incidence matrix to provide solutions to two multi-agent network control problems. In particular, we first considered the problem of state agreement with quantized communication in continuous systems, and then looked into the problem of distance-based formation control. In both cases, stabilizing control laws were provided for the case of tree graphs. This topology is directly related to the null space of the incidence matrix, thus making its role evident in these cases of networked control problems.

\section{References}

Arcak, M. (2007). Passivity as a design tool for group coordination. IEEE Transactions on Automatic Control, 52(8), 1380-1390.

Baillieul, J., \& Suri, A. (2004). Information patterns and hedging Brocketts theorem in controlling vehicle formations. In 43rd IEEE conf. decision and control (pp. 556-563).

Cao, M., Anderson, B., Morse, A., \& Yu, C. (2008). Control of acyclic formations of mobile autonomous agents. In 47 th IEEE conf. on decision and control (pp. 1187-1192).

Carli, R., Fagnani, F., \& Zampieri, S. (2006). On the state agreement with quantized information. In 17 th Intern. symp. networks and systems (pp. 1500-1508).

Cortes, J., Martinez, S., \& Bullo, F. (2006). Robust rendezvous for mobile autonomous agents via proximity graphs in arbitrary dimensions. IEEE Transactions on Automatic Control, 51(8), 1289-1298.

Fax, J., \& Murray, R. (2002). Graph Laplacians and stabilization of vehicle formations. In 15th IFAC world congress.

Godsil, C., \& Royle, G. (2001). Springer Graduate Texts in Mathematics: Vol. \# 207. Algebraic graph theory.
Guattery, S., \& Miller, G. (2000). Graph embeddings and laplacian eigenvalues. SIAM Journal of Matrix Analysis and Applications, 21(3), 703-723.

Hendrickx, J., Anderson, B., \& Blondel, V. (2005). Rigidity and persistence of directed graphs. In 44th IEEE conf. decision and control (pp. 2176-2181).

Horn, R. A., \& Johnson, C. R. (1996). Matrix analysis. Cambridge University Press.

Johansson, K. H., Speranzon, A., \& Zampieri, S. (2005). On quantization and communication topologies in multi-vehicle rendezvous. In 16th IFAC world congress (electronic proceedings).

Kashyap, A., Basar, T., \& Srikant, R. (2007). Quantized consensus. Automatica, 43(7), 1192-1203.

Lygeros, J., Johansson, K., Simic, S., Zhang, J., \& Sastry, S. (2003). Dynamical properties of hybrid automata. IEEE Transactions on Automatic Control, 48(1), $2-17$.

Olfati-Saber, R., \& Murray, R. (2004). Consensus problems in networks of agents with switching topology and time-delays. IEEE Transactions on Automatic Control, 49(9), 1520-1533.

Olfati-Saber, R., \& Shamma, J. (2005). Consensus filters for sensor networks and distributed sensor fusion. In 44th IEEE conference on decision and control (pp. 6698-6703).

Zelazo, D., Rahmani, A., \& Mesbahi, M. (2007). Agreement via the edge laplacian. In 46th IEEE conference on decision and control (pp. 2309-2314).

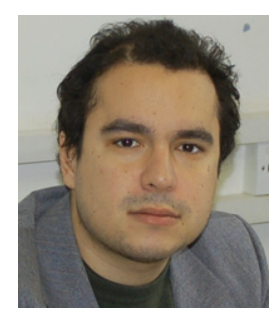

Dimos V. Dimarogonas was born in Athens, Greece in 1978. He received the Diploma in Electrical and Computer Engineering in 2001 and the Ph.D. in Mechanical Engineering in 2006, both from the National Technical University of Athens (NTUA), Greece. From May 2007 to February 2009, he was a Postdoctoral Researcher at the Automatic Control Laboratory, KTH ACCESS Linnaeus Center, School of Electrical Engineering, Royal Institute of Technology (KTH), Stockholm, Sweden. Dr. Dimarogonas was one of the four recipients of the KTH ACCESS Linnaeus Center Award for best post-doctorial application, selected among more than one hundred candidates. Since February 2009, he is a Postdoctoral Associate at the Laboratory for Information and Decision Systems, Massachusetts Institute of Technology (MIT), Cambridge, MA, USA. His current research interests include Multi-Agent Systems, Robot Navigation, Networked Control and EventTriggered Control. He is a member of the Technical Chamber of Greece.

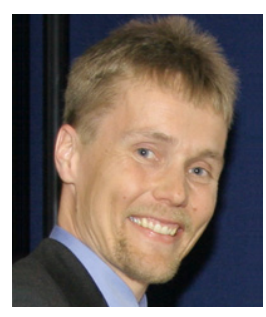

Karl H. Johansson is Director of the ACCESS Linnaeus Centre and Professor at the School of Electrical Engineering, Royal Institute of Technology, Sweden. He is a Wallenberg Scholar and holds a Senior Researcher Position with the Swedish Research Council. He received M.Sc. and Ph.D. degrees in Electrical Engineering in 1992 and 1997 from Lund University in Sweden. He has held visiting positions at UC Berkeley (1998-2000) and California Institute of Technology (2006-07). His research interests are in networked control systems, hybrid and embedded control, and control applications in automotive, automation and communication systems. He is the Chair of the International Federation of Automatic Control (IFAC) Technical Committee on Networked Systems since 2008. He has served on the IEEE Control Systems Society Board of Governors and on the Executive Committees of the European research projects HYCON and RUNES. He is on the editorial boards of the IEEE Transactions on Automatic Control and the IET Control Theory \& Applications, and previously of the IFAC journal Automatica. He was awarded a six-year Individual Grant for the Advancement of Research Leaders from the Swedish Foundation for Strategic Research in 2005. He received the triennial Young Author Prize from IFAC in 1996 and the Peccei Award from the International Institute of System Analysis, Austria, in 1993. He received Young Researcher Awards from Scania in 1996 and from Ericsson in 1998 and 1999. 